\title{
PROPUESTA DE BASE DE DATOS Y CATÁLOGO DE MOSAICOS TARDOANTIGUOS EN LA HISPANIA ROMANA.
}

\author{
BEATRIZ GARRIDO RAMOS \\ Universidad Nacional de Educación a Distancia
}

\begin{abstract}
Resumen
Desde hace años en nuestro país se asiste a la falta de interés en lo que respecta al ámbito de la musivaria romana. En consecuencia, se ha detectado una precaria puesta en valor y un insuficiente proceso de conservación, ambas cuestiones inadecuadas para poder garantizar el correcto mantenimiento de nuestro patrimonio cultural, de gran riqueza pero altamente desconocido por la sociedad. Es probable que ante la falta de información no seamos conscientes de la riqueza ni del alto grado de abandono al que se encuentra expuesto desde hace décadas. Por ello, se optó por realizar una base de datos y un catálogo sobre mosaicos romanos tardoantiguos, que pretende seguir creciendo en los próximos años, evitando de esta forma condenarse al olvido, o lo que resultaría fatal, al deterioro y consiguiente desaparición, como ha sucedido ya con algunos de ellos.

Palabras clave
\end{abstract}

Base de datos, catálogo, Hispania, Humanidades Digitales, investigación, mosaicos romanos tardoantiguos, Open Access/Open Education.

\section{PROPOSAL OF DATABASE AND CATALOGUE OF MOSAICS LATE ANTIQUITY IN ROMAN HISPANIA.}

\section{Abstract}

For years in our country are attending the lack of interest in regards to the scope of the Roman out-dated. Consequently, detected a precarious value and an inadequate process of conservation, both issues of inadequate to ensure proper maintenance of our heritage cultural, great wealth but highly unknown by the society. It is likely that, in the absence of information, we are not aware of wealth or high grade of abandonment that is exposed for decades. Therefore it was decided to perform a database and a catalogue of Roman mosaics late antiquity, intends to continue growing in the coming years, thus preventing condemned to oblivion, or what would be fatal, the deterioration and subsequent disappearance, as It has already happened with some of them.

\section{Keywords}

Database, catalogue, Hispania, Digital Humanities, research, Roman mosaics late antiquity, Open Access/Open Education. 
Tal y como expone el profesor Javier Andreu Pintado: "Es evidente que el nuevo entorno mediático - - y tecnológico - que, actualmente, se abre ante quienes trabajamos en la investigación en patrimonio - $-\mathrm{y}$, por tanto, en Humanidades - ofrece extraordinarias oportunidades para la difusión, la divulgación, la comunicación y la promoción de nuestros trabajos como medio para garantizar la comprensión, la educación y el disfrute de los receptores de esos contenidos, verdadero reto y colofón de la gestión del patrimonio. Hacer posible esa correspondencia entre transmitir y sensibilizar, entre enseñar y promocionar, entre formar y motivar — casi conforme a los principios auriseculares del docere, delectare, monere - exige, desde luego, altas capacidades de trabajo, una cierta pericia -que puede complementarse con formación específica- en manejo del lenguaje, de los medios y de los registros digitales y, sobre todo, fe e ilusión en el trabajo que se realiza"1.

El patrimonio cultural peninsular es sin duda elevado y de incalculabe valor. Sirva de ejemplo el ámbito de los mosaicos que es el área de estudio del presente trabajo. El gran volumen de datos existente relativo a la musivaria de la Península Ibérica en época romana nos condujo a replantear la necesidad de diseñar una herramienta que permitiese aglutinar y gestionar de manera sencilla y eficaz toda esa información, de forma que se adaptara a determinados estándares para que aumentara su utilidad y facilitara la recuperación de dicha información, y cumpliera igualmente su cometido de difusión social y puesta en valor de un patrimonio cultural que con frecuencia no es lo suficientemente tenido en cuenta, o al menos no tanto como mereciera.

A colación de lo anterior, Carmen Ladrón de Guevara expuso la siguiente reflexión sobre la catalogación del patrimonio cultural español: "En España contamos con una larga tradición de catalogación del patrimonio cultural. Aunque los primeros atisbos de catalogación los podemos encontrar a fines del siglo XVIII es en el siglo XX cuando tiene lugar el primer intento serio de catalogación de la riqueza patrimonial, el "Catálogo Monumental de España" de 1900, que aunque no llegó a concluirse, estableció una metodología que sirvió de modelo para catálogos posteriores. Con la creación y promulgación de las sucesivas leyes de protección del patrimonio, los intentos por realizar unos catálogos efectivos y prácticos se multiplicaron, aunque sus resultados fueron muy diferentes y dispares. Merece la pena destacar la gran labor realizada por el "Servicio Nacional de Información Artística, Arqueológica y Etnográfica", que desde los años sesenta hasta los ochenta emprendió una encomiable actividad de catalogación, en la que participaron diversos departamentos de las Universidades españolas. En el último cuarto de siglo y debido a los cambios políticos acaecidos en España y a la consolidación del Estado de las Autonomías, el número de catálogos e inventarios se ha diversificado y, aunque el número de bienes catalogados se ha incrementado considerablemente, estos siguen siendo escasos si se tiene en cuenta el conjunto total del patrimonio cultural español"2.

La temática de la investigación se ha centrado en la musivaria existente en las villas tardoantiguas de la Península Ibérica en época romana. Una labor fundamental al respecto ha sido la recuperación de textos, y la unificación y clasificación de todo ese material existente hasta la fecha, destacando entre otros textos los conocidos Corpus de Mosaicos Romanos de España (CMRE), obra iniciada por D. Antonio García y Bellido, que conforman sin duda una importantísima fuente de conocimiento para toda persona interesada en el ámbito de la musivaria, puesto que su objetivo es el estudio y la publicación de todos los mosaicos del Imperio. En este sentido, la visión del profesor D. José María Blázquez (director del CMRE entre 1976 y 1997) fue decisiva, ya que hasta entonces, y en contraste con otros países de gran tradición investigadora y otras disciplinas, los mosaicos romanos en España apenas habían suscitado interés más allá de su clasificación entre las artes menores.

\footnotetext{
1 Andreu, 2014: 37.
}

2 Ladrón, 2007: 1. 


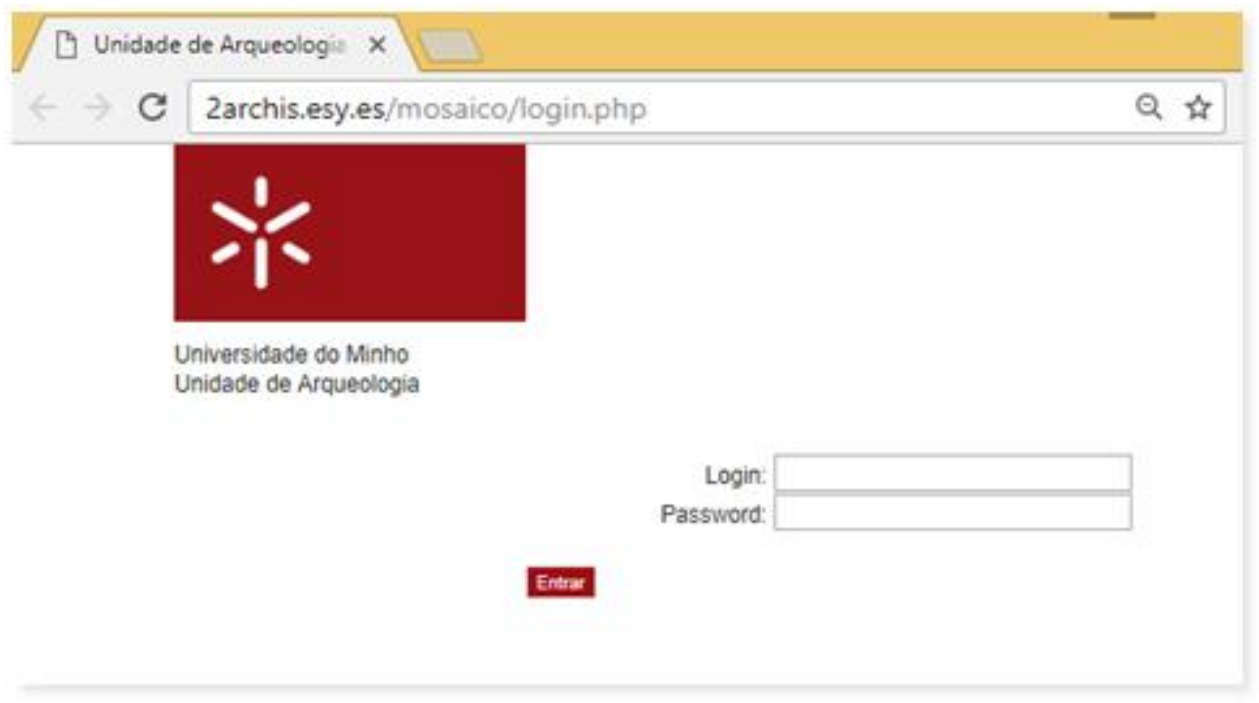

Fig.1. Acceso al Back Office. Fuente: Archivo personal de la autora.

Tradicionalmente se ha considerado que "el mosaico ha sido una fuente de conocimiento fundamental para la reconstrucción del contexto socio-cultural del que participaban los antiguos propietarios hispano-romanos, latifundistas de carácter oligárquico o altos funcionarios de la administración imperial’3.

\section{Musivaria romana desde la perspectiva de las humanidades digitales y open access.}

Hasta la fecha se han llevado a cabo diversos proyectos ${ }^{4}$ a nivel nacional e internacional relativos al ámbito de la musivaria. También existen recursos de difusión como el portal de cultura http://geo.spainisculture.com/index.php/es En nuestro caso, el presente texto parte del trabajo expuesto en el II Congreso Internacional de Humanidades Digitales Hispánicas celebrado en la UNED de Madrid en octubre de 2015, bajo el título "Mosaicos en la Hispania romana y Humanidades Digitales" y posteriormente de la investigación llevada a cabo por la autora durante su tesis doctoral "Iconografía musivaria en la Peninsula Ibérica en época romana: investigación y difusión desde el campo de las Humanidades Digitales".

En primer lugar, sería conveniente definir el concepto de "Humanidades Digitales", ¿qué se entiende por ello? Muchos autores como Kirschenbaum ${ }^{6}$, Mc Carthy o Moretti ${ }^{7}$, por citar a algunas de las grandes voces de las HD, se han referido a ello. La Dra. Gimena del

${ }^{3}$ Matthews, 1975: 13-25 citado en Bermejo, 2007: 41.

${ }^{4}$ Fuentes o proyectos digitales que ya han trabajado sobre el tema de la musivaria dentro y fuera de España, por citar algunos ejemplos, son:

http://www.asprom.org/resources/links.html

http://m.sussex.ac.uk/byzantine/mosaic/

https://www.wga.hu/frames-e.html?/html/zgothic/mosaics/9/index.html

https://www.archesproject.org/what-is-arches/

http://www.seemosaics.org/

${ }^{5}$ Este artículo presenta un extracto del trabajo realizado por la autora durante su estancia en la Unidade de Arqueologia de la Universidade do Minho (Braga, Portugal), con motivo de la concesión de una beca otorgada por la Facultad de Geografía e Historia de la UNED. Se diseñó una herramienta y se realizó una base de datos y un mapa con todas las villas romanas con mosaicos, de forma que permitiera la navegación por la iconografía de la tardoantigüedad en España (Hispania) y Portugal. Posteriormente, se procedió a la correspondiente clasificación de cada uno de los mosaicos que formaban parte de la base, mediante la aplicación de un lenguaje documental que permitiera trabajar con estándares internacionales.

6 Véase: https://mkirschenbaum.files.wordpress.com/2011/03/ade-final.pdf

7 Autor que estudia grandes corpus de textos y se refiere en su discurso a conceptos como el de la cuantificación, visualización y la lectura distante (Distant Reading). 
Río por su parte expone que las "Humanidades Digitales se erigen como una transdisciplina global que atraviesa a las Humanidades con una mirada desde lo digital, sin dejar de lado la capacidad de adecuarse a las particularidades culturales, sociales y económicas de un contexto académico determinado". Pero la respuesta podría traducirse desde luego en tantas definiciones como formas de trabajar existentes en el ámbito de las Humanidades. Por ese motivo, simplemente nos limitaremos a esbozar una breve definición que consideramos representa una parte importante del trabajo realizado por la autora en su tesis y que se desarrollará brevemente en páginas sucesivas. Nos referimos a la siguiente definición: "Son un conjunto de herramientas tecnológicas que permiten la globalización del conocimiento extendido, dando la posibilidad de acceder a gran cantidad de información aplicando una metodología estandarizada. Conducen por tanto a la investigación y difusión de diversas disciplinas"”.

Méndez Martínez alude a conceptos como "herramientas tecnológicas", "metodología estandarizada" y "investigación y difusión". A todos ellos añadiremos igualmente el término que hemos denominado "cultura conectada" y que pretende mostrar la necesidad de conectar de una forma homogénea, accesible y estandarizada la información y toda la investigación realizada. Buena prueba de ello es el movimiento "Open Access/Open Education"10. Es lógico pensar consecuentemente que "una apertura documental permitirá un gran abanico de posibilidades hasta ahora impensable. Los partidarios de los datos abiertos argumentan que las limitaciones van en contra del bien común, y que los datos, tienen que ponerse a disposición del público"11.

Igualmente, también es sumamente importante el nuevo abanico de posibilidades que han brindado no solo las Humanidades Digitales, sino también las TIC y los programas específicos para el tratamiento y visualización de la información o para la realización de estadísticas entre otros. A colación de esto, y según expone Rincón Zamorano: "El tratamiento digital de la información ha permitido simplificar y automatizar muchas tareas que anteriormente se realizaban manualmente, con el consiguiente coste tanto de recursos materiales como humanos. Con el acceso a una mayor cantidad de información, tanto en cantidad como en variedad, surgen nuevos problemas para su tratamiento, siendo necesarios nuevos métodos y tecnologías. La informática se encarga de definir estos procedimientos y tecnologías que nos facilitan el manejo de grandes volúmenes de información de una manera amigable" ${ }^{\prime 2}$.

\section{Breve introducción al concepto de "villae"y "domus".}

Después de la introducción anterior también se torna necesario tratar brevemente los conceptos de "villae" y "domus", así como realizar una aproximación a ellos con el fin de contextualizar la investigación. Previamente hay que exponer a este respecto que las fuentes antiguas y clásicas son fundamentales para el estudio de aspectos históricos, políticos, sociales, artísticos y geográficos entre otros.

8 Véase la definición completa en TaDiRAH - Taxonomía sobre Actividades de investigación digital en Humanidades: http://www.vocabularyserver.com/tadirah/es/index.php?tema=61\&/humanidades-digitales

${ }^{9}$ Méndez, 2015: 24.

${ }^{10}$ En nuestro caso, la propuesta que se realiza se trata de una base de datos en Acceso Abierto, lo que está más cerca de un recurso educacional abierto. Para ampliar información sobre el concepto Open Access/Open Education véase: https://sparcopen.org/

${ }^{11}$ Garrido, 2017b: 16.

12 Rincón, 2015: 2. 


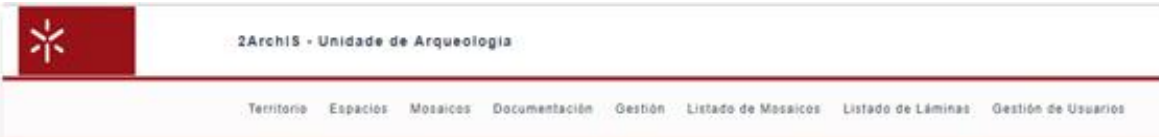

Fig. 2. Menú principal del Back Office. Fuente: Archivo personal de la autora.

La evolución histórico-artística que se sucedió en la Península Ibérica en época romana fue, probablemente y en gran medida, consecuencia del desarrollo de la cultura indígena anterior (cultos, deidades, etc.), que como es lógico, dejaría restos de su existencia, tanto en lo referente a cuestiones geográficas (evolución del paisaje) como en el arte en general y en la iconografía romana en particular.

En primer lugar, como decíamos, para contextualizar el contenido existente en la base de datos y catálogo antes mencionados, hay que exponer que los ejemplos recopilados y estudiados se tratan tanto de yacimientos, villae y domus, como de mosaicos aislados, cuya ubicación de estos últimos por el momento desconocemos, y por lo tanto, no se tiene claro si pudieron situarse en una vivienda o en otro lugar.

Según Jorge Carracedo, "las villae se suelen definir como asentamientos rurales de explotación agrícola-ganadera situados en llanuras, y en las que se distingue por una banda la explotación agrícola y por otra, la residencia del señor o propietario. Aparecen siempre ligadas a la proximidad de las vías de comunicación"13.

En cuanto a las domus, que es como se conoce a un tipo de casa romana, se trataba de la vivienda de las familias con un cierto nivel económico y poder adquisitivo, al frente de la cual se situaba el dominus (pater familias o cabeza de familia). De este tipo de vivienda existen buenos ejemplos en Pompeya y Herculano.

\section{Villas tardoantiguas en la Península Ibérica.}

A continuación se expondrán algunas cuestiones generales sobre las villas tardoantiguas en la Península Ibérica. Pero antes, debe recordarse cuál era la situación peninsular en el momento histórico que nos ocupa. Teniendo en cuenta los estudios de Tovar y Blázquez: "Con el fin de las guerras cántabras y la reorganización administrativa de Hispania comienza una nueva era. La Península, pacificada, pierde sus rasgos culturales propios, salvo en aquellas regiones del norte y noroeste, donde la situación de atraso los mantiene más largo tiempo. La uniformadora romanización se va extendiendo por todas partes y al acontecer histórico, centrado en Roma, no se manifiesta en las provincias pacificadas sino en la actividad administrativa, desempeñada por funcionarios que enseguida empiezan a profesionalizarse" ${ }^{\prime 4}$.

Por su parte, tal y como expuso José María Blázquez: "Con la crisis del siglo III y durante el Bajo Imperio, al disminuir la presión de Roma, renacen una serie de técnicas y motivos decorativos de gran tradición indígena" principal de las villas tardorromanas es el de la continuidad con la etapa anterior"16.

\footnotetext{
${ }^{13}$ Carracedo, 2013.

14 Tovar/Blázquez, 1975: 122.

15 Blázquez, 1978: 251.

16 Díaz/Martínez/Sanz, 2007: 251.
} 


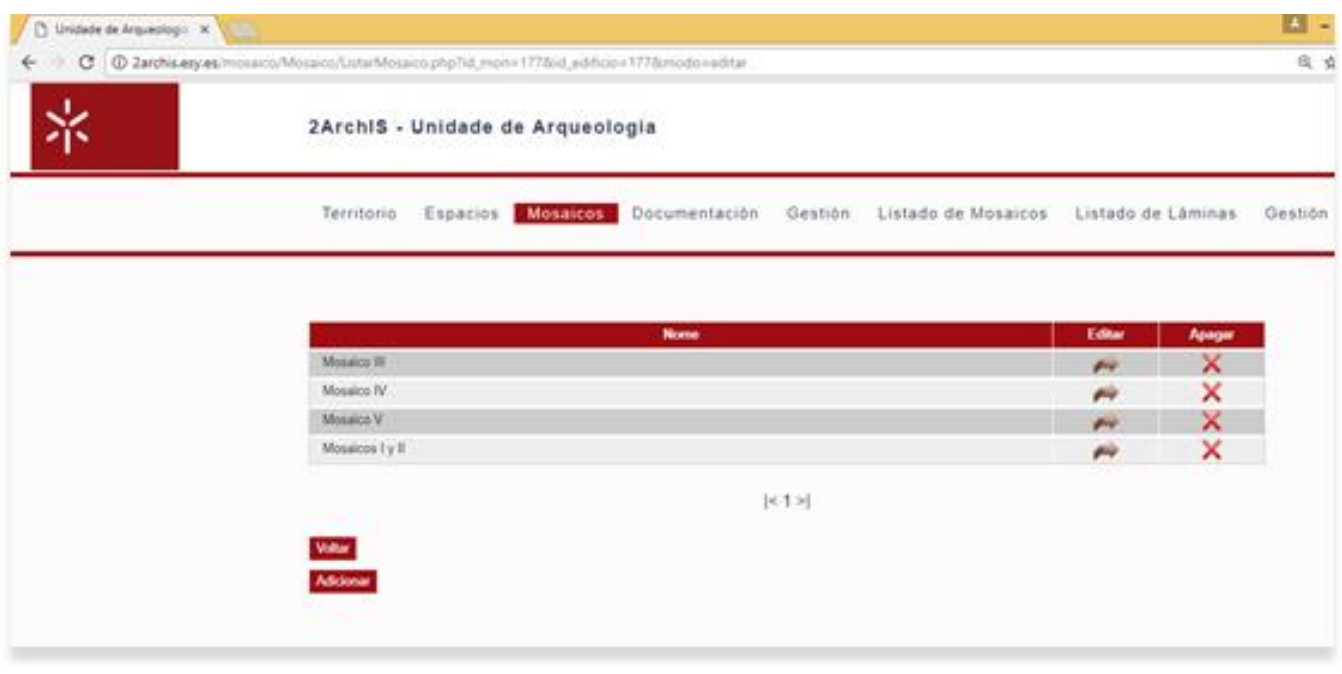

Fig. 3. Mosaicos asociados al espacio de $A$ Cigarrosa. Fuente: Archivo personal de la autora.

Autores como Ariño y Díaz por su parte aluden a que "el siglo IV puede considerarse como un "siglo de Oro" para el campo hispano, caracterizado por el esplendor de las villae y su estabilidad productiva. Estos autores, junto a J. Arce y G. Ripoll, también han destacado la intensísima relación económica y social existente entre la ciudad y el campo... La máxima expresión de la arquitectura rural romana es la villa" ${ }^{" 17}$.

En lo referente a las villas y mosaicos, hay que destacar que su estudio permite explorar el contexto histórico del momento, sobre todo si tenemos presente afirmaciones como las que realizan Tovar y Blázquez: "Las luchas continuadas interrumpieron la administración, destruyeron la vida económica, cortaron el comercio y la industria y empeoraron considerablemente la mala situación económica de los estratos más bajos de la población. Las villas del bajo Imperio fueron arrasadas en su casi totalidad en estos años, como se deduce del hecho de que, salvo alguna, como la de la Cocosa (Badajoz), no hay prueba ninguna de que fueran habitadas después de los primeros años del siglo $\mathrm{V}$, y en muchas hay huellas de destrucciones violentas por fuego. En la Península tan sólo se conocen dos o tres mosaicos que se puedan datar con seguridad en el siglo $\mathrm{V}$, todo lo cual indica bien el hundimiento de la economía del siglo anterior. Hacia el año 445 Salviano de Marsella ${ }^{18}$ habla de la total barbarización de Hispania; incluso cesaron los juegos del circo y las representaciones teatrales ${ }^{19}$. Aunque puede haber mucho de exageración en el predicador, sus afirmaciones responden sin duda a un fondo de verdad"20.

Teniendo en cuenta todas estas cuestiones, entendemos que las villas constituyeron el principal motor de la economía hispanorromana del momento que nos ocupa. Este punto hizo que se llevara a cabo un replanteamiento por parte de la autora ante la detección de la insuficiente labor realizada hasta la fecha en el campo de la musivaria romana en Hispania, cuestión que sin duda necesita de una nueva aplicación de métodos y técnicas que favorezcan su difusión y contribuyan a la puesta en valor de este rico patrimonio.

Finalmente, por lo que respecta a la tipología de villae, las villas romanas en Hispania adoptaron variantes de planificación diversas. Si atendemos como criterio clasificatorio de las villas al de la disposición en planta y el modelo constructivo empleado en el edificio principal de la misma, se observan dos tradiciones constructivas muy marcadas:

\footnotetext{
${ }_{17}$ Díaz/Martínez/Sanz, 2007: 251.

18 De gub. Dei V 5, 23.

19 Salv. De gub. Dei VI 7, 38; 8, 39.

20 Tovar/ Blázquez, 1975: 157-158.
} 
- Modelo mediterráneo: con planta central organizada en torno a un peristilo, que organiza todo el edificio.

- Modelo nórdico o céltico: con planta rectangular, con corredor en la fachada, muchas veces con presencia de torres a ambos lados que delimitan patios en los modelos más elaborados. Ejemplo significativo de este último tipo de villa lo constituye la villa romana de Noville (Mugardos, A Coruña).

Fernández de Castro ${ }^{21}$, por su parte, estableció las siguientes tipologías de villa:

- De plan diseminado: dentro de esta tipología de villa encontramos dos modos de disposición de las edificaciones:

- Sin un orden aparente.

- Con alineación a lo largo de un espacio abierto rectangular: de este tipo derivan a su vez las villas en forma de "U" o "L", más generalizadas en el norte de Europa

- Urbano-rústica: la disposición más usual en el área del Mediterráneo fue la centralizada en torno a un peristilo, sin embargo, también hay muchos ejemplos de villas que, conforme a una estructuración estrictamente funcional y rural, aplicaron a las cámaras de habitación y departamentos termales los requisitos decorativos de la casa en la ciudad observándose una ausencia del patio porticado ${ }^{23}$.

- Residencial: esta tipología de villa puede subdividirse en cuatro grupos ${ }^{24}$ :

1. Este primer grupo el desarrollo arquitectónico y ornamental de las habitaciones de la villa desvelan el carácter señorial del dominus.

2. En este grupo estarían presentes aquellas mansiones residenciales que por su limitación documental y arqueológica, permite desvelar su condición señorial debido a que se reduce únicamente al núcleo de la mansión, sin descartar la existencia de un sector servil y agrícola.

3. El tercer grupo reúne a villas de apariencia señorial aisladas geográficamente y de tipología arquitectónica indeterminada, cuya característica principal es la ausencia de peristilo.

4. El último grupo se corresponde con aquellas villas donde lo único descubierto son las termas al no haberse realizado una excavación completa de la villa.

- Marítima: las villas romanas de Hispania no responden a la realidad del prototipo de la misma, ya que estas villas se caracterizan por tener un xystus y un barrio marítimo. Sin embargo, hay un aspecto que si tiene en común una de las villas hispánicas con las características de una villa marítima: la apertura al mar mediante un pórtico, como es el caso de la villa de Centroña (Puentedeume, La Coruña).

- Con establecimiento rústico: villas en las que únicamente se conocen los espacios de explotación agraria o industrial ya que, debido a la falta de datos arqueológicos, no se conocen las habitaciones de residencia ${ }^{25}$.

Otra clasificación que puede establecerse dentro de la tipología de villa es la que alude a la distribución espacial de la parte residencial. De esa forma, se encuentran las siguientes viviendas:

- De planta alargada.

\footnotetext{
${ }^{21}$ Fernández, 1982: 61.

${ }^{22}$ Fernández, 1982: 64.

${ }^{23}$ Fernández, 1982: 69-70.

${ }^{24}$ García/Ruiz, 2004: 210-211.

25 García/Ruiz, 2004: 212.
} 


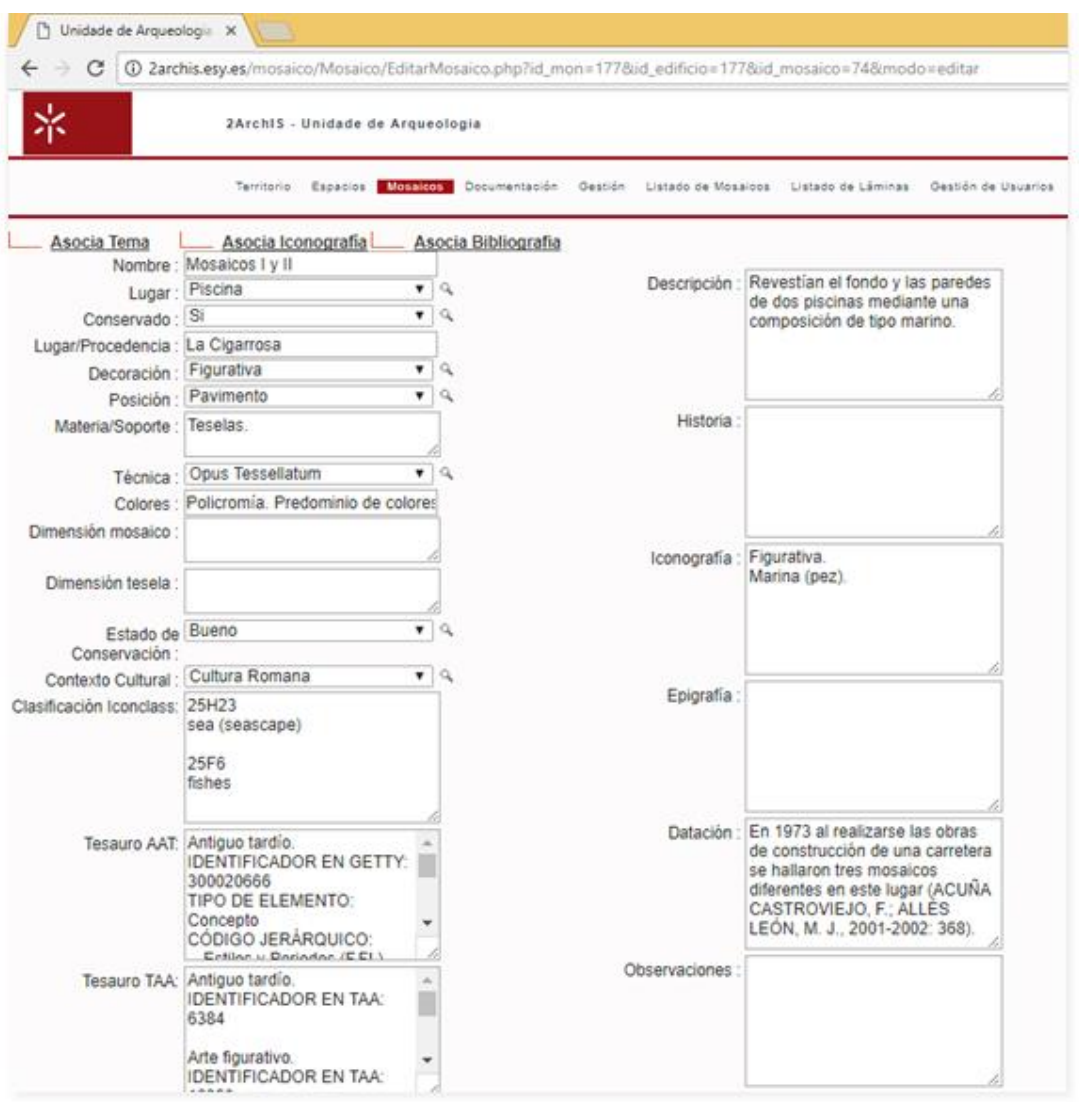

Fig. 4. Información de los Mosaicos I y II de A Cigarrosa. Fuente: Archivo personal de la autora.

- De peristilo: las “villae de peristilo" es el tipo más extendido en la Península Ibérica. Por ese motivo, se analizarán y extraerán conclusiones al respecto de las villas y las de corredor y de planta diseminada, que se encuentran en una proporción mucho menor. Se subdivide en tres dependiendo del tipo de peristilo y la función que se le otorga al mismo como $^{26}$ :

- Espacio ajardinado rodeado de pórticos.

- Patio rodeado de pórticos, donde el jardín es omitido a favor de un patio porticado enlosado de tipología helénica.

- Peristilo doble: dentro de este tipo de vivienda podemos encontrar dos variantes como son:

- Casa de atrio: en la zona hispánica se presenta como una unidad menor dentro de una edificación doméstica con un espacio central donde se ubica el atrio.

- Casa de atrio y peristilo: continuación del modelo anterior pero con la introducción de una columnata de origen helenístico.

Por último, en cuanto a la funcionalidad de los espacios dentro de la villa se refiere, se podría hablar de:

- Espacios de habitación: triclinio, oecus, cubicula, etc. En el caso de los cubicula, Fernández Castro expone que "en ocasiones el acceso no era directo y babia que llegar a una habitación a través de otra. Su función más directa era la de dormitorio"27.

- Espacios serviles y agrícola-industriales: de utilidad doméstica y con finalidad agrícola ${ }^{28}$.

\footnotetext{
26 García/Ruiz, 2004: 215-216.

${ }^{27}$ Fernández, 1982: 209-210.

${ }^{28}$ Fernández, 1982: 217-219.
} 
Arasa expone que ambas funciones, residencial y agropecuaria son, por lo tanto, convergentes $^{29}$.

\section{Aproximación metodológica para el estudio de los mosaicos romanos en Hispania.}

El marco teórico-metodológico que tomaremos como punto de partida será la "Arquitectura de Paisaje" (ArqPa) y los conceptos que la autora ha considerado oportuno denominar "Arquitectura del Mosaico" (ArqMo) y "Arqueología del Mosaico" (ArMo), como una nueva vía que pretende una aproximación a la arquitectura desde el estudio de los vestigios arqueológicos y el análisis de la cultura material musivaria existente en la Península Ibérica en época romana.

Nuestra aproximación a la arquitectura se producirá desde la disciplina de la Historia del Arte, y concretamente desde la Arqueología, con el análisis de la cultura material de la época que nos ocupa, así como de la Iconografía, desde el estudio iconográfico y mitológico de las representaciones llevadas a cabo en los mosaicos.

El ámbito de la investigación, como se expone, se circunscribe geográficamente a la Hispania romana. Por el momento se han repertorizado un buen número de villas, pero la intención es abarcar todas las villas tardías pertenecientes a dicho periodo. Se han tenido en cuenta los trabajos realizados hasta el momento, los cuales se centraban en zonas geográficas específicas. Por ello, "la aportación principal ha sido la recuperación de textos, y la unificación y clasificación de todo ese material existente hasta la fecha, destacando entre otros textos los Corpus de Mosaicos de España que conforman sin duda una importantísima fuente de conocimiento para toda persona interesada en el ámbito concreto de la musivaria. No obstante, además de los corpus, se han tomado como referencia los estudios de un buen número de autores como Blázquez, Lancha, Morand, Chavarría y Fernández Castro entre otros, que previamente habían realizado estudios sobre mosaicos en diversas zonas de España"30.

\section{Propuesta de herramienta, base de datos y catálogo para los mosaicos romanos.}

A continuación, se mostrará un extracto del trabajo realizado por la autora en la Unidade de Arqueologia de la Universidade do Minho (Braga, Portugal), durante la que se diseñó una herramienta y se realizó una base de datos y un mapa con las villas romanas con mosaicos, de forma que permitiera la navegación por la iconografía de la tardoantigüedad en España y Portugal. Posteriormente, se procedió a la clasificación de cada uno de los mosaicos que formaban parte de la base, mediante la aplicación de un lenguaje documental que permitiera trabajar con un estándar internacional como es el caso de Iconclass, utilizado en trabajos de iconografía e Historia del Arte.

Por lo que respecta al diseño de la herramienta para realización de la base de datos para mosaicos, nos referimos concretamente al Sistema de Información 2 ArchIS de la base de datos diseñada con la colaboración de la ingeniera Natàlia Botica en la Unidad de Arqueología de la Universidade do Minho. Las principales características de la herramienta son las siguientes:

- Se diseñó y desarrolló una aplicación de Back Office implementada en PHP y HTML para el registro de los datos correspondientes a los apartados que denominamos "Territorio" (municipio o distrito), "Espacio" (yacimiento, villa, domus, termas, necrópolis), "Mosaicos" (geométricos, figurativos o ambos), y a la asociación de "Temas" (cacería, mitología, acuático, etc.), "Iconografía", "Imágenes" y "Bibliografía".

${ }^{29}$ Arasa, 2003: 161.

30 Garrido, 2017a: 82-83. 


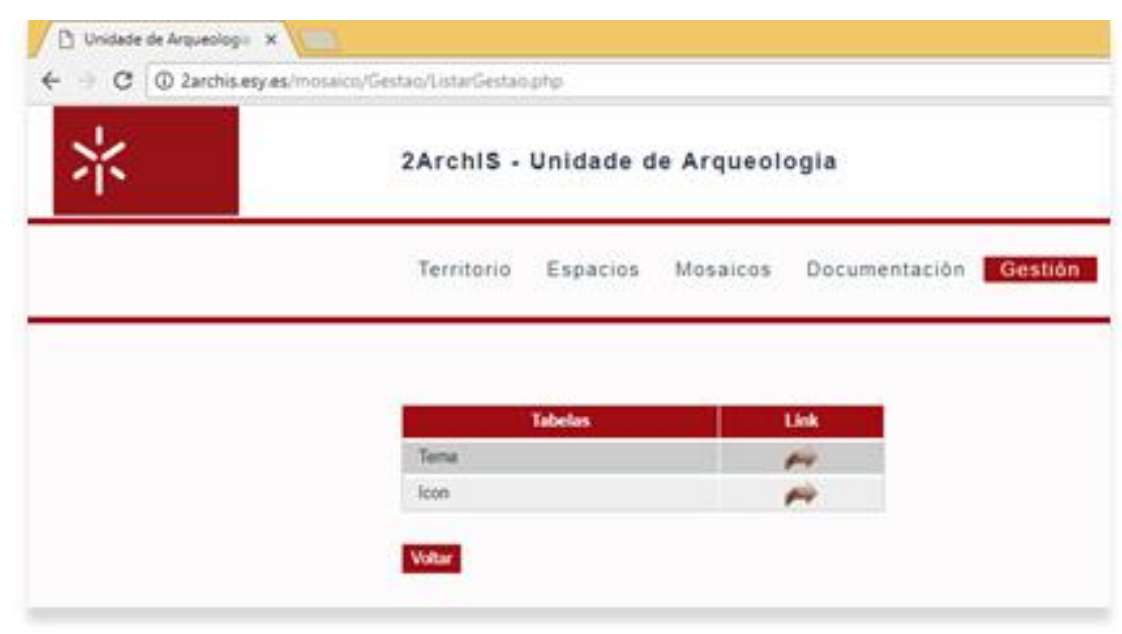

Fig. 5. Menú para el registro del tema y la iconografía asociados a los mosaicos. Fuente: Archivo personal de la autora.

- Durante la investigación se aplicaron Sistemas de Información Geográfica (SIG), que ayudan a visualizar, comprender e interpretar la realidad, haciendo comprensibles y manejables los datos, y permitiendo establecer patrones y tendencias a través de mapas, gráficos o informes. La gestión geográfica de la información es esencial para comprender la historia y los sucesos que la acompañan, contribuyen a una mejor comprensión y por lo tanto, facilitará la toma de decisiones en relación a ello. Se utilizó un Sistema de Información Geográfica (SIG) para el análisis geográfico y de distribución espacial de los "Territorios" y "Mosaicos", genérica y por "Temas" e "Iconografía", realizada con la aplicación ArcGis Map de la ESRI ${ }^{31}$, estableciendo una relación automática a la base de datos MySQL, permitiendo aplicar Queries, es decir realizar búsquedas de información.

- La Georreferenciación (coordenadas X, Y) se tomó de Google Map.

El registro digital de la información obtenida durante el proceso de investigación se realizó en una aplicación de Back Office en la que se incorporaron e integraron los datos resultantes del estudio de las villas tardoantiguas tales como texto, fotografías, plantas de villas, planos, reconstrucciones 3D y vídeos, así como documentación en formato pdf para su posterior descarga. La estructura de la aplicación, desarrollada de forma integrada y modular, se implementa con herramientas open source y de interfaz web. (Fig. 1) (Fig. 2)

El sistema fue diseñado siguiendo la Norma CIDOC-CRM ${ }^{32}$. El Modelo de Referencia Conceptual (CRM) es una ontología formal aplicada en el ámbito del Patrimonio Cultural, llevado a cabo por un grupo de trabajo del Comité de Documentación que se desarrolla en el Consejo Internacional de Museos (ICOM). Como se expone en la web del CIDOC: "Con el fin de aprovechar plenamente el potencial del CRM como un medio que permite el intercambio de información e integración en la comunidad de museos y más allá, CIDOC decidió en Londres 1999 presentar la CRM con la norma ISO para la normalización... El CIDOC CRM fue aceptado como borrador de trabajo por la norma ISO / TC46 / SC4 /

31 Tal y como se expone en la web http://www.esri.es/ se trata de una empresa especializada en el desarrollo de Sistemas de Información Geográfica. Su misión es inspirar cambios positivos en la sociedad a través del uso de la ciencia y los SIG (o GIS en terminología anglosajona). ESRI apuesta por el Cloud haciendo de la transformación digital una realidad.

32 "Working Groups". En: <http://network.icom.museum/cidoc/working-groups/overview/> [01/07/2017]. 
WG9 en septiembre de 2000. El 12 de septiembre de 2006 se hizo oficial norma ISO 21127: $2006^{333 "}$.

El CIDOC CRM es la culminación de más de 10 años de trabajo del Grupo de Trabajo de Normas para la Documentación CIDOC y CIDOC CRM SIG. Desde el 9/12/2006 es la norma oficial ISO 21127: 2006. En Diciembre de 2014 se presentó una nueva versión basada en la versión 5.0.4 del CIDOC CRM, la ISO 21127: 2014. A colación de este extracto, es importante destacar que el Grupo de Trabajo de Normas para la Documentación del CIDOC ha desarrollado una declaración de principios de documentación para los museos ${ }^{34}$ (aprobado en la Asamblea General Anual 2012 del CIDOC celebrada en Helsinki), y además, trabaja en diversas cuestiones entre las que se encuentra el fomento de vocabularios controlados y recursos terminológicos específicos de relevancia para museos, como es el caso de Iconclass ${ }^{35}$, clasificación internacional que se ha aplicado a los mosaicos que conforman nuestra base de datos.

\section{Menú “mosaicos”, “gestión” y “documentación”}

Como se ha comentado anteriormente, existen diversos menús en la aplicación diseñada, pero nos centraremos, a modo de ejemplo, en los que se han denominado menú "Mosaicos", menú "Gestión" y menú "Documentación" por resultar especialmente significativos dentro del proceso. En este apartado de la aplicación denominado menú "Mosaicos" se encuentran los distintos mosaicos asociados a cada territorio y espacio que forma parte de la base de datos. Se puede observar a continuación el ejemplo del territorio A Rúa, cuyo espacio, el yacimiento de A Cigarrosa, tiene asociados cinco mosaicos. (Fig. 3)

En la siguiente imagen se puede ver la información relativa a dos de esos mosaicos, concretamente los denominados I y II. (Fig. 4)

Mediante el menú "Gestión" se pueden insertar el "Tema" o los temas (según su representación) y los "Iconos" (palabras clave) que se asocian a los mosaicos, pudiendo con ambas opciones especificar la iconografía que aparece representada en ellos. (Fig. 5). Algunos de los temas a seleccionar son arquitectura, cacería, circo, epigrafía, fauna, figura humana, flora, geometría, mitología, etc. Por lo que respecta a las palabras clave, para su selección se ha recurrido a los motivos destacados del mosaico, de forma que al realizar un listado con dichas palabras se facilita la descripción de las escenas, debido a que se asegura que se procede de igual forma para cada elemento y se recurre a seleccionar la misma palabra (en nuestro caso siempre masculino y singular) con el fin de facilitar a posteriori las búsquedas y garantizar unos resultados óptimos del funcionamiento de la base de datos. De igual forma, se permite la creación de nuevas palabras clave por si en un futuro fuese necesario recurrir a palabras que actualmente no se encontraran registradas.

\footnotetext{
33 Para ampliar información sobre la norma ISO 21127:2006 véase "Information and documentation -A reference ontology for the interchange of cultural heritage information". En: $<$ https://www.iso.org/standard/34424.html> [15/06/2017].

34 Tal y como se expone en la web del CIDOC: "El grupo de trabajo tiene como objetivo actuar como un punto de enlace con los órganos e iniciativas relevantes que desarrollan las normas de relevancia para la documentación de los museos. Ejemplos de tales cuerpos son el Text Encoding Initiative, CCO y MICHAEL. Los miembros del grupo están trabajando en una asignación de TEI a la CIDOC CRM. En el área de control de la terminología, el grupo tiene previsto revisar los marcos técnicos, tales como las normas de tesauro actualizadas de NISO y la British Standards Institution, SKOS y Topic Maps".

35 A la hora de trabajar con imágenes es una de las opciones que puede barajarse ya que permite gestionar vocabularios controlados para la descripción o clasificación de iconos o figuras, tal y como se expone en la web del proyecto: <http://www.iconclass.nl/home> Otros ejemplos son Getty Research Institute $<$ http://www.getty.edu/research/tools/vocabularies/aat/> (vocabularios), Pelagios $<$ http://pelagiosproject.blogspot.com.es/> (que permitirá visualizar en un mapa dicho vocabulario), Ariadne $<$ http://www.ariadneinfrastructure.eu/ $>$ (que reúne e integra las infraestructuras de datos de investigación arqueológica existente), y Perseus < http://www.perseus.tufts.edu/hopper/> (proyecto de biblioteca digital de la Universidad Tufts que reúne una colección digital de fuentes para Humanidades). (Garrido, 2017b: 21).
} 


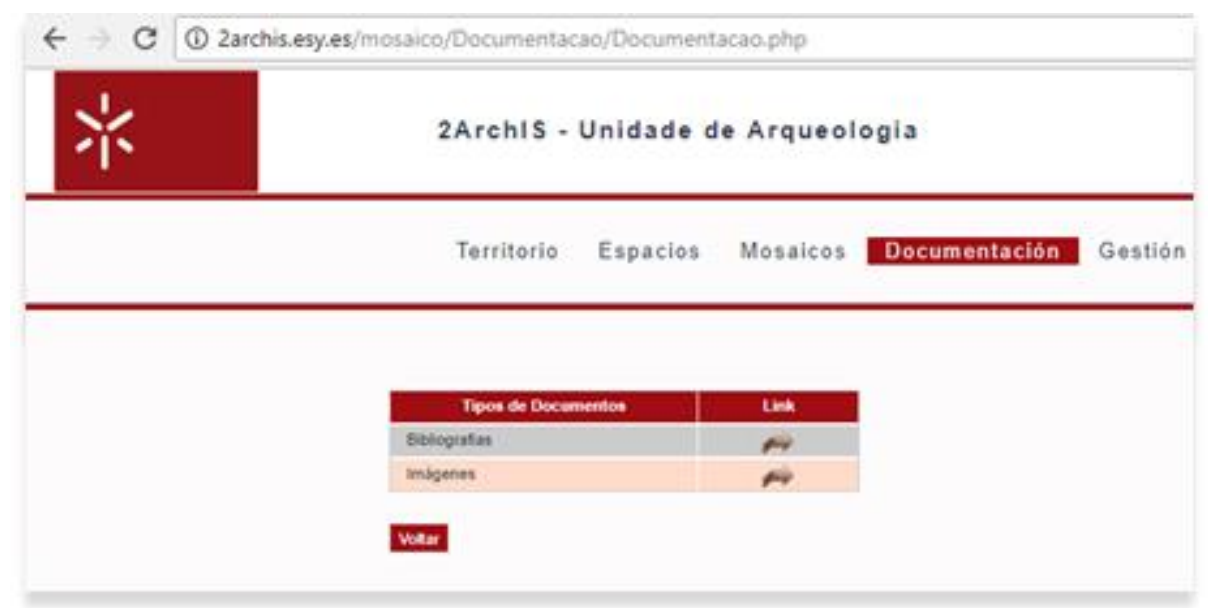

Fig. 6. Menú Documentación. Fuente: Archivo personal de la autora.

En el menú "Documentación" se puede acceder a la "Bibliografía" y a las "Imágenes" asociadas a los mosaicos. En este ejemplo, en color rojo aparecen seleccionadas las imágenes. (Fig. 6). Posteriormente se selecciona la imagen desde nuestro ordenador, se sube a la aplicación y se cumplimenta la siguiente información relativa, en este caso, a los Mosaicos I y II. (Fig. 7). Una de las principales ventajas de realizar el proceso de esta forma es que al quedar almacenada la imagen en nuestra base de datos no existe la posibilidad de que en un futuro pueda desaparecer o no estar visible, como sucede con frecuencia cuando se indica una URL que posteriormente no se encuentra o ha cambiado de dirección. De esta forma, aseguramos que el contenido esté siempre disponible y actualizado.

Llegados a este punto conviene explicar que la selección de los campos que conforman el menú "Mosaicos" se ha debido a dos cuestiones básicas: la primera, porque se ha tomado como referencia la web del Museo Arqueológico Naciona $\hat{p}^{6}$, y la segunda, porque se observó que en gran medida los campos seleccionados se ajustaban al estándar Dublin $\mathrm{Core}^{37}$, otra de las cuestiones que se ha trabajado especialmente durante la investigación con la intención de obtener una información que facilitase su inserción en otros proyectos, instituciones museísticas y el tratamiento de la misma para investigadores y profesionales del campo de la musivaria.

36 Véase: <http:// ceres.mcu.es>.

37 Tal y como se expone en la propia web de Dublin Core Metadata Iniatiative: "The Dublin Core Metadata Element Set is a vocabulary of fifteen properties for use in resource description. The name "Dublin" is due to its origin at a 1995 invitational workshop in Dublin, Ohio; "core" because its elements are broad and generic, usable for describing a wide range of resources. The fifteen element "Dublin Core" described in this standard is part of a larger set of metadata vocabularies and technical specifications maintained by the Dublin Core Metadata Initiative (DCMI). The full set of vocabularies, DCMI Metadata Terms [DCMI-TERMS], also includes sets of resource classes (including the DCMI Type Vocabulary [DCMI-TYPE]), vocabulary encoding schemes, and syntax encoding schemes. The terms in DCMI vocabularies are intended to be used in combination with terms from other, compatible vocabularies in the context of application profiles and on the basis of the DCMI Abstract Model [DCAM]. All changes made to terms of the Dublin Core Metadata Element Set since 2001 have been reviewed by a DCMI Usage Board in the context of a DCMI Namespace Policy [DCMI-NAMESPACE]. The namespace policy describes how DCMI terms are assigned Uniform Resource Identifiers (URIs) and sets limits on the range of editorial changes that may allowably be made to the labels, definitions, and usage comments associated with existing DCMI terms". $<$ http://dublincore.org/documents/dces/> [05/05/2017]. 
Fig. 7. Imagen asociada a los Mosaicos I y II de A Cigarrosa. Fuente: Archivo personal de la autora.
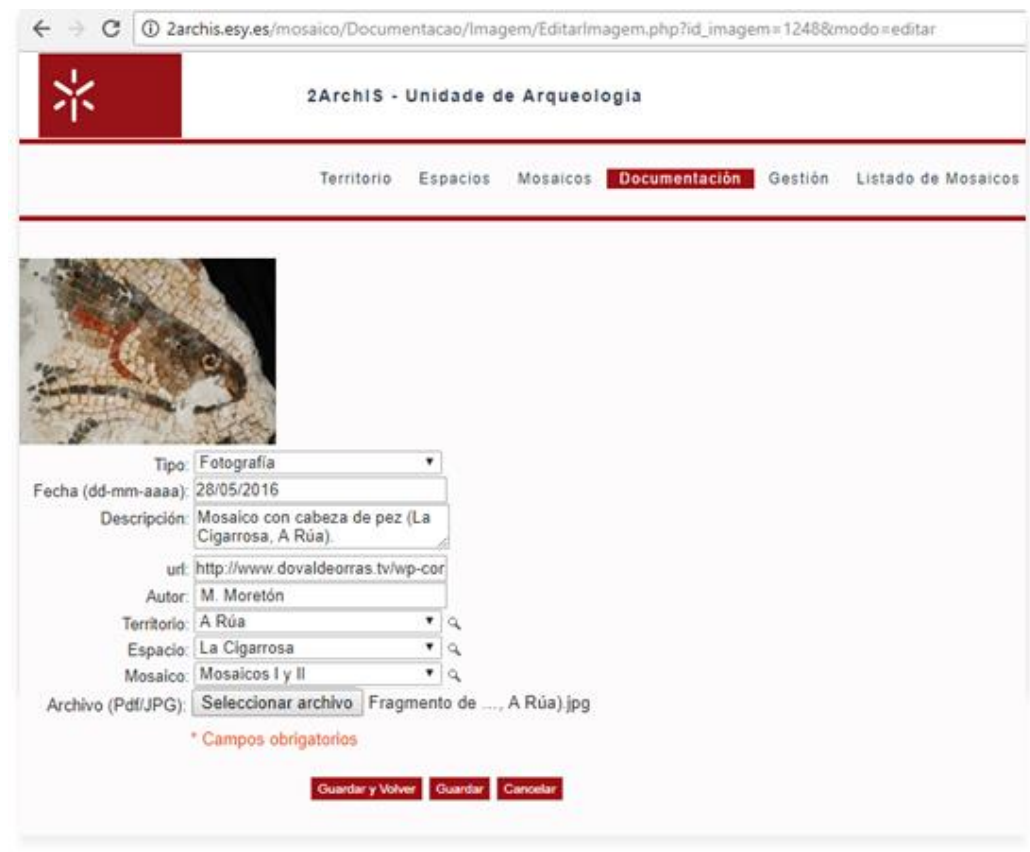

Durante el proceso de investigación llevado a cabo durante la creación de la base de datos y la clasificación de los mosaicos se optó por utilizar el estándar Dublin Core en otras cuestiones porque posee elementos de descripción que otorgan un significado semántico a los mismos. Así se obtiene una homogeneidad que facilitará la interoperabilidad y contribuirá a la asociación de vocabularios controlados, como sucedió en nuestro caso por ejemplo con la aplicación de Iconclass y el Tesauro AAT del Getty Museum así como la realización de una Taxonomía denominada TaxMOS HD (abreviatura de Taxonomía,

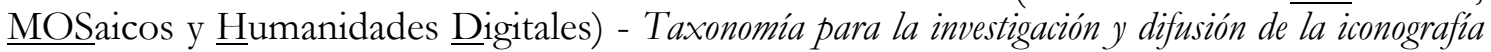
musivaria romana desde las Humanidades Digitales, que se puede consultar en línea en el siguiente enlace: http://www.vocabularyserver.com/taxmos/

Cabe recordar que según Abed Gregio et al. una Taxonomía "es un proceso científico (o un sistema particular) de categorizar entidades, es decir, de organizarlas en grupos. Un sistema taxonómico debe ser claro y consistente, flexible, exhaustivo y práctico" 38 . Igualmente, debido a la extensión que tomó la investigación, se optó por la realización de una Taxonomía con la finalidad de ordenar jerárquicamente los conceptos que formaban parte de nuestra base de datos de forma que permitiera hacerse una idea más clara del contenido de la misma. Tal y como expone Centelles al respecto, "aplicando el principio etimológico, la taxonomía se ha definido como la ciencia que trata de los principios, métodos y fines de la clasificación, y procura la organización jerarquizada y sistemática, dando nombres a grupos de elementos y a los elementos mismos. Una taxonomía es un tipo de vocabulario controlado en que todos los términos están conectados mediante algún modelo estructural (jerárquico, arbóreo, facetado, etc.) y especialmente orientado a los sistemas de navegación, organización y búsqueda de contenidos” de los sitios web ${ }^{39}$.

\section{Conclusiones}

La muestra que conforma el repertorio de mosaicos de nuestra base de datos, lejos de quedarse aquí, se trata de un work in progress, un punto de partida, puesto que nuestra intención es la de contribuir lo máximo posible al estudio de la musivaria tardoantigua romana en Hispania, de forma que pueda seguir aumentando progresivamente, contando

38 Abed Gregio et al., 2007 citado en Díaz/Joyanes/Medina, 2009: 245.

${ }^{39}$ Centelles, 2005 citado en Díaz/Joyanes/Medina, 2009: 245. 
para ello con la colaboración de toda persona interesada en el ámbito de la iconografía musivaria romana en nuestro país. Tanto la herramienta diseñada como todo el trabajo en sí se encontrará a disposición de los usuarios que serán los que nos ayuden a evaluar la propuesta metodológica presentada en la tesis de la autora, y que en consecuencia, contribuyan a la mejora de todo el material y la actualización del mismo, con la finalidad de que podamos construir un verdadero mapa de la musivaria de época romana, que por otra parte, sería extensible a todo el imperio.

Como se ha expuesto previamente, se ha recurrido a la utilización de los Sistemas de Información Geográfica (SIG), los cuales llevan tiempo formando parte de la investigación arqueológica y favorecen por ello el inventario, el tratamiento espacial de la información, la gestión y el desarrollo de la propia investigación en cuanto al estudio del patrimonio cultural se refiere.

Posteriormente, se procedió a la correspondiente clasificación de cada uno de los mosaicos que formaban parte de la base, mediante la aplicación de un lenguaje documental que permitiera trabajar con un estándar internacional como es el caso de Iconclass, utilizado en trabajos de iconografía e Historia del Arte, complementado a su vez con el tesauro $A A T$ del Getty y la información referente a la localización de cada territorio, llevado a cabo con GeoNames. Se trata por tanto de un ejemplo de trabajo interdisciplinar (puesto que como se observará en páginas sucesivas, una parte de nuestro trabajo se aproxima a la rama de la informática) desarrollado en el campo de la musivaria romana en particular, y de la Arqueología, la Geografía, la Historia e Historia del Arte y las Humanidades Digitales en general. No obstante, el siguiente paso será la creación de una herramienta de front office para dar visibilidad a los datos recopilados y permitir así realizar búsquedas de acuerdo con determinados criterios con el fin de extraer estadísticas sobre los datos de los mosaicos repertorizados.

Finalmente, hay que destacar el impacto de la cultura digital que ha tenido lugar en los últimos años, lo cual ha contribuido al desarrollo de nuevas formas de trabajo, a la aplicación de nuevas metodologías y a la inserción de otras herramientas que se adapten y permitan seguir investigando e incluso perfeccionando los resultados obtenidos durante el proceso. En relación a ello, Paul Spence (2014) se ha referido entre otros términos al concepto de la e-Research enfocado al trabajo colaborativo así como a la cibercultura. Sin duda, la sociedad actual - que se ha denominado a sí misma como "sociedad de la información" - requiere de un proceso de adaptación y asimilación de esas nuevas formas y métodos de trabajo, y para ello, las ya conocidas como Humanidades Digitales, surgidas en parte de la "informática humanística", contribuirán en gran medida a la consecución de ese objetivo en todas las disciplinas, siendo las Humanidades un ámbito en el que como en nuestro caso resulte especialmente interesante. 


\section{Bibliografía}

Andreu, J.: "Arqueología en directo: canales de comunicación y transferencia de resultados en la investigación sobre patrimonio arqueológico: la ciudad romana de 'Los Bañales' (Uncastillo, Zaragoza)". En: Baraibar, Álvaro (ed.): Visibilidad y divulgación de la investigación desde las Humanidades Digitales. Experiencias y proyectos. Pamplona: Universidad de Navarra, Colección BIADIG, Publicaciones Digitales del GRISO, (2014), pp. 17-41.

Arasa, F.: "Las villas: explotaciones agrícolas". En: Gozalbes, Manuel/Albiach, Rosa/Bonet, Helena (coords.): Romanos y visigodos en tierras valencianas. Valencia: Museu de Prehistòria de València, (2003), pp. 161-166.

Bermejo, J.: “Análisis Contextual de la Iconografía de Aquiles en los Mosaicos Hispanos de Época Bajoimperial”. En: Actas del VI Encuentro de Jóvenes Investigadores, Historia Antigua, Universidad Complutense de Madrid, (2007), pp. 41-52.

Blázquez, J. M.: Historia económica de la Hispania romana. Madrid: Ediciones Cristiandad, S. L. (1978).

Botica, N./Martins, M.: "Sistemas de informação em Arqueologia - A experiência de Bracara Augusta". En: Congreso Internacional de Arqueoloxia de Vilalba, Lugo, 11-14 junio. (2008).

Botica, N.: Contributo do Sistema de Informação 2ARCHIS para o conhecimento das cidades Romanas de Braga e Lugo. Colección PHILTÁTE. (2017).

Díaz, F. N./Joyanes, L./Medina, V. H.: “Taxonomía, ontología y folksonomía, ¿qué son y qué beneficios u oportunidades presentan para los usuarios de la web?". En: Univ. Empresa, 8, 16, Bogotá (Colombia), (2009), pp. 242-261.

Díaz, P./Martínez, C./ Sanz, F.: Historia de España V. Historia Antigua. Hispania tardoantigua y visigoda. Madrid: Istmo. (2007).

Dublin Core: Dublin Core Metadata Iniatiative. En: $<$ http://dublincore.org/documents/dces/> [05/05/2017].

ESRI España: ArcGIS. En: <http://www.esri.es/> [05/05/2017].

Fernández, M. C.: Villas romanas en Hispania. Madrid. (1982).

García, R./Ruiz, A.: "Aproximación al estudio de las villas romanas en España. Las villas de Albacete, Ciudad Real y Toledo". En: Arse: Boletín anual del Centro Arqueológico Saguntino, 38, (2004), pp. 201-240.

Garrido Ramos, B.: "Mosaicos en la Hispania Romana y Humanidades Digitales". En: II Congreso Internacional de Humanidades Digitales Hispánicas: Innovación, globalización e impacto, UNED, Madrid, 5-7 de octubre. (2015). En: <http://espacio.uned.es/congresosuned/index.php/hdh2015/hdh2015/paper/view/132>

[01/08/2015].

Garrido Ramos, B.: "Iconografía musivaria en la Hispania romana: Iconclass, un estudio de caso desde la perspectiva de las Humanidades Digitales". En: ArtyHum Revista Digital de Artes y Humanidades, 38, sección de Investigación, Vigo, (2017a), pp. 80-95. En: $<$ https://www.artyhum.com/revista/38/mobile/index.html\#p=80> [01/09/2017].

Garrido Ramos, B.: "Visualización de datos y clasificación Iconclass: un estudio de caso desde la perspectiva de las Humanidades Digitales". En: Caracteres. Estudios culturales y críticos de la esfera digital, 6, 1, Salamanca, (2017b), pp. 10-33. En: $<$ http:// revistacaracteres.net/revista/vol6n1 mayo2017/iconclass> [05/08/2017].

ISO 21127:2006: A reference ontology for the interchange of cultural heritage information. En: $<$ https://www.iso.org/standard/34424.html> [15/06/2017]. 
Kirschenbaum, M. G.: "What Is Digital Humanities and What's It Doing in English Departments?". En ADE BULLETIN, 150. (2010) En: $<$ https://mkirschenbaum.files.wordpress.com/2011/03/ade-final.pdf> [25/04/2018].

Ladrón de Guevara, C.: "La catalogación del patrimonio cultural: conceptos generales". En: Seminario sobre la planificación de inventarios en centroamérica, San Salvador, 21-25 de mayo. (2007).

"Los mosaicos de A Cigarrosa. Historia de un hallazgo (Museo Arqueológico Provincial de Orense)". En: Carracedo, J.: "Sobre la presencia romana en la comarca de Valdeorras". En: Historia y Arqueología, 6 de noviembre. (2013).

Méndez, J. Á.: "Definición de Humanidades Digitales". En: ArtyHum Revista Digital de Artes y Humanidades, Monográfico de Humanidades Digitales: La realidad de las Humanidades Digitales en España y América Latina, 1, Vigo, (2015), p. 24.

En: <https://www.artyhum.com/monograficos/hd/\#/24> [01/11/2015].

Red Digital de Colecciones de Museos de España. En: <http://ceres.mcu.es> [01/08/2015].

Rincón Zamorano, M.: "Introducción a la visualización de la información en Humanidades Digitales". En: Curso de Experto Profesional en Humanidades Digitales. UNED. T. 1. (2015) p. 2.

Río Riande, G. del: TaDiRAH - Taxonomía sobre Actividades de investigación digital en Humanidades. (2014)

En: <http://www.vocabularyserver.com/tadirah/es/index.php?tema=61\&/humanidadesdigitales $>$ [25/04/2018].

Spain is Culture: Portal de la Cultura. España es Cultura. En: $<$ http://geo.spainisculture.com/index.php/es> [25/04/2018].

SPARC: Advancing Open Access, Open Data, Open Education. En: <https://sparcopen.org/> [25/04/2018].

Spence, P.: "Prólogo: La investigación en Humanidades Digitales en el mundo hispano". En: Romero, Esteban/Sánchez, María (eds.): Ciencias Sociales y Humanidades Digitales: técnicas, herramientas y experiencias de e-Research e investigación en colaboración. Tenerife: Sociedad Latina de Comunicación Social, (2014), pp. 9-12.

Tovar, A./Blázquez, J. M.: Historia de la Hispania romana. Madrid: Alianza editorial. (1975). 